\title{
Large airway complications in COVID-19 pneumonia
}

\author{
James May', Nordita Ramos-Bascon², Natalie Barnes², Brendan Madden² \\ ${ }^{1}$ Department of Respiratory Medicine, St George's University Hospital NHS Foundation Trust, London; ${ }^{2}$ Department \\ of Cardiothoracic Medicine, St George's University Hospital NHS Foundation Trust, London, UK
}

\begin{abstract}
COVID-19 pneumonia can cause respiratory failure which requires specialist management. However, the inflammatory nature of the condition and the interventions necessary to manage these patients such as endotracheal intubation and tracheostomy can lead to large airway pathology which may go unrecognised. We describe five of the $44(11 \%)$ consecutive patients referred to our specialist ARDS team between April and June 2020 with confirmed COVID-19 pneumonia who developed diverse large airway pathology which comprised of: supraglottic oedema, tracheal tear, tracheal granulation tissue formation, bronchomalacia, and tracheal diverticulum. Large airway pathology may be underappreciated in severely ill patients with COVID-19 pneumonia and
\end{abstract}

Correspondence: Professor Brendan Madden, Department of Cardiothoracic Medicine, St George's University Hospital NHS Foundation Trust, London SW17 0QT, UK.

E-mail: brendan.madden@stgeorges.nhs.uk

Key words: COVID-19; trachea; bronchi; pathology.

Contributions: JM, BM, conception and design, analysis and interpretation of data, drafting the article or revising it critically for important intellectual content; NRB, NB, analysis and interpretation of data, drafting the article. All the authors have read and approved the final version of the manuscript and agreed to be accountable for all aspects of the work.

Conflict of interest: The authors declare that they have no competing interests, and all authors confirm accuracy.

Ethics approval and consent to participate: Not applicable.

Consent for publication: Consent to publish material obtained from all patients involved.

Availability of data and material: The data used to support the findings of this study are available from the corresponding author upon request.

Received for publication: 22 April 2021.

Accepted for publication: 13 July 2021.

${ }^{\circ}$ Copyright: the Author(s), 2021

Licensee PAGEPress, Italy

Monaldi Archives for Chest Disease 2022; 92:1894

doi: 10.4081/monaldi.2021.1894

This article is distributed under the terms of the Creative Commons Attribution Noncommercial License (by-nc 4.0) which permits any noncommercial use, distribution, and reproduction in any medium, provided the original author(s) and source are credited. should be considered in patients with unexplained air leak, prolonged need for mechanical ventilatory support, and repeated failed extubation or decannulation. If suspected, such patients should be managed by a team with expertise in large airway intervention and early specialist advice should be sought.

\section{Introduction}

COVID-19 pneumonia caused by the SARS-CoV-2 virus is now well recognised for the respiratory illness it can cause due to effects on the lung parenchyma and pulmonary vasculature [1]. Diffuse alveolar damage and resultant acute respiratory distress syndrome as well as heightened risk of pulmonary emboli (PE) and the formation of in situ thrombosis can contribute to profound hypoxia due to ventilation/perfusion (VQ) mismatch and such patients are frequently intubated and mechanically ventilated.

Clinician focus on gas exchange and haemodynamic parameters in these critically unwell patients is an unquestionable priority as we improve our understanding of the disease. However, large airway pathology may be underappreciated as a result. These patients are at increased risk of complications in the trachea and main bronchi not only due to the direct effect of the virus on respiratory epithelium but also due to interventions such as endotracheal intubation and tracheostomy.

Our tertiary centre has experience not only in the management of high numbers of critically ill patients with COVID-19 pneumonia but also in large airway intervention and act as a referral centre for London and the South East. 44 critically unwell patients with confirmed COVID-19 pneumonia were referred to our Acute Respiratory Disease Service (ARDS) team for assessment from its inception in April 2020 until June 2020 [2]. This was led by a Professor of Cardiothoracic and Intensive Care Medicine with expertise in rigid and interventional bronchoscopy. We present five of these who had associated large airway pathology.

\section{Case \#1}

A 61-year-old male with a background of hypertension and type two diabetes (T2DM) was intubated for type one respiratory failure (T1RF). He was extubated on to non-invasive ventilation after ten days (with two intervening failed extubations). Six days following extubation, he deteriorated with sepsis and was re-intubated. Six days later, a surgical tracheostomy was performed. There was a persistent cuff leak and he was decannulated and retintubated with use of GlideScope which demonstrated supraglottic and glottic oedema. The following day a revision surgical tracheostomy was performed with insertion of a larger tube (8.0 
Portex). This was initially successful but then required high cuff pressures to prevent air leak. It was changed a third time to a size 9.0 adjustable flange cuffed tube and positioned with the aid of bronchoscopy. This once again confirmed the presence of supraglottic and glottic oedema. His admission was complicated by bacteraemia (hence a decision was made not to treat his airway pathology with parental corticosteroid), PE with right ventricular strain, an asystolic cardiac arrest, and a gastrointestinal bleed. He died on the fourth week of his admission.

\section{Case \#2}

A 69-year-old male with a background of hypertension and hypercholesterolaemia was admitted to his local hospital. On day 13 he required emergency intubation on the ward for worsening T1RF despite continuous positive airway pressure (CPAP). The procedure was described as "difficult". A bougie was not used. The following day surgical emphysema was appreciated around the neck. Computed-tomography (CT) chest confirmed the presence of extensive surgical emphysema, pneumomediastinum, and pneumoperitoneum without an obvious underlying cause. A right sided pneumothorax became apparent on chest radiograph the following day. Bilateral surgical chest drains were inserted and placed on suction but his surgical emphysema worsened. Repeat CT imaging suggested a small posterior tracheal perforation so he was transferred to our unit. Rigid and fibreoptic bronchoscopy demonstrated a $1 \mathrm{~cm}$ defect on the left posterolateral tracheal wall with surrounding inflammatory change suggesting an original defect of 2-3 cm. A tracheostomy was subsequently fashioned and placed over the tear with bronchoscopic guidance. His wean was ventilation was prolonged with successful decannulation after six weeks. His admission was additionally complicated by ventilator associated pneumonia, pericarditis, and cardiac arrhythmia requiring permanent pacemaker insertion. The tracheal tear healed spontaneously and he was successfully discharged and remains under surveillance with our team.

\section{Case \#3}

A 47-year-old male was admitted to his local hospital where he was intubated for T1RF following failed CPAP therapy (described as "easy intubation", with use of GlideScope). He had no past medical history of note. He was transferred for venovenous extracorporeal membrane oxygenation which he received for a 22-day period. During this period a percutaneous tracheostomy was performed. He was transferred to our unit for ongoing care and ventilation wean. He was noted to have persisting cough with high secretion burden and episodic desaturation. In addition, he had a markedly weak voice when the tracheostomy cuff was deflated and a speaking valve applied. Rigid and fibreoptic bronchoscopy was performed prior to attempting decannulation. This demonstrated a proximal rim of granulation tissue above the site of entry of the tracheostomy tube producing a $30-40 \%$ local stenosis. There was no distal granulation tissue or secretions. This was not severe enough to prevent decannulation which was performed successfully 48 hours later (approximately five weeks after the initial percutaneous tracheostomy). He was subsequently discharged to a rehabilitation unit for ongoing care and remains under our continued surveillance.

\section{Case \#4}

A 63-year-old male was transferred from a local hospital where he had been intubated for T1RF. Twelve days later he was transferred to our unit and underwent percutaneous tracheostomy on day sixteen. His past medical history was significant for T2DM, hypertension, asthma, and obesity. There were issues with tenacious secretions and flexible bronchoscopy was performed for microbiological sampling and airways clearance. Aspergillus fumigatus was isolated from bronchoalveolar lavage. He developed left lower lobe collapse and pleural effusion which required intercostal chest drain insertion. He was treated with antifungals and antibiotics and a further flexible bronchoscopy showed dynamic left main bronchomalacia. A stent was deployed in the left main bronchus and he was successfully decannulated. He was then discharged to a rehabilitation unit with follow up by our team.

\section{Case \#5}

A 53-year-old male was transferred from a local hospital where he had been intubated. He had impaired glucose tolerance and asthma. Two days after intubation, a thoracic CT scan confirmed the presence of pneumomediastinum. A percutenaous tracheostomy was performed 12 days after intubation. He also had acute kidney injury requiring haemofiltration, type two myocardial injury, bacterial sepsis, and PE. His ventilation improved and his tracheostomy was removed five weeks after admission. Due to septic deterioration, he underwent whole body CT. This demonstrated findings consistent with acute pyelonephritis as well as a gas-distended tract with overlying granulation tissue at the tracheostomy site consistent with a tracheal diverticulum. This was confirmed on bronchoscopy and a conservative approach was taken. He remained asymptomatic, made good progress and was repatriated to his local hospital. He remains under our prospective surveillance.

\section{Discussion}

We present five patients with varied large airway pathology with confirmed COVID-19 pneumonia who required critical care admission, endotracheal intubation, and tracheostomy.

Large airway - including supraglottic, glottic, and subglottic oedema is a recognised complication of endotracheal intubation, with frequency increasing with duration of intubation [3]. The average time from endotracheal intubation to tracheostomy in COVID-19 is not insignificant and has been reported to be 19.7 days in one cohort study [4]. Since the virus can be associated with large airway inflammation [5] upper airway oedema should be considered as a reason for failed extubation or persisting signs of upper airway obstruction following extubation in these patients.

Tracheal injury secondary to intubation has been well described, with emergency intubation being a risk factor [6] with some cases described in COVID-19 [7] including three spontaneous cases attributed to coughing [8]. In our patient, we suspect that the tear was present from the initial intubation. The index of suspicion of this diagnosis should be high, especially in cases of unexplained surgical emphysema and pneumomediastinum.

In our third patient, prolonged need for tracheostomy likely 
resulted in granulation tissue formation proximal to the site of tracheostomy entry. This may be missed as bronchoscopy is often performed via the tracheostomy for ease and patient comfort but this only allows for distal airway assessment. Formation of proximal granulation tissue may cause serious airways obstruction and secretion accumulation and should be identified and treated prior to decannulation if suspected [9].

Tracheobronchomalacia is a historically well described late complication of intubation and tracheostomy [10]. This may contribute to failed extubation or failed tracheostomy removal. Temporary airway stenting may be an option for some patients.

Our final patient had a tracheal diverticulum. Prior imaging confirmed this was not congenital. The inflammatory nature of COVID-19 and the need for prolonged endotracheal intubation or tracheostomy are presumed contributory factors. The condition is yet to be described in association with COVID-19.

We identified 5 (11\%) of the 44 consecutive patients referred to our ARDS team in our tertiary centre over a three-month period who had significant large airway pathology requiring specialist intervention. We did not observe a noticeable difference in the presentation, diagnostics, or treatment approach when we have drawn comparisons to patients treated through our unit prior to the pandemic [11]. The incidence, however, was higher than that seen in our usual pre-pandemic practice and we feel this is likely to be a reflection of the increase in numbers of critically ill patients requiring airway intubation and intervention as a result of infection with COVID-19. The true incidence is as yet unknown.

To further this, we have seen a proportion of critically ill COVID patients requiring high levels of positive pressure ventilation thus subjecting the airways and lung parenchyma to the consequences of barotrauma. Secondly, the use of the corticosteroid dexamethasone is now routine in the majority of patients following the publication of the RECOVERY trial [12] and steroid therapy may be a contributor to delayed airway healing. In addition, many patients are also being treated for concurrent venothromboembolism with anticoagulation, which again may affect healing but may also impair visibility at the time of intubation if there is active or recent bleeding.

Intubation may also have been performed by less experienced - albeit competent - operators who were doing so under extremes of time pressure given the potential for rapid clinical deterioration in COVID-19 pneumonia. One would anticipate an increased reliance on a bougie in these settings and this may be contributing, especially in the case of laryngeal tears. Laryngeal tears affecting the membranous trachea are well described in association with endotracheal intubation [13] and although considered safe there are case reports in the non-Covid literature describing laryngeal damage with bougie use $[14,15]$.

We postulate that these factors either in isolation or combined are contributing to the observed pathology and the higher number of patients encountered.

\section{Conclusions}

Important learning points we have drawn from these cases is that not only is there diversity in the large airway pathology possi- ble in patients with Covid-19 but it may also be under appreciated as the focus has largely been on the management of ventilation and haemodynamic support. In cases of unexplained surgical emphysema, prolonged need for or difficult mechanical ventilatory support, and repeated failed extubation or decannulation, the index of suspicion of large airway pathology should be heightened. In this setting, involvement of a tertiary centre or a team experienced in large airway intervention should be sought early.

\section{References}

1. Wiersinga W, Rhodes A, Cheng AC et al. Pathophysiology, transmission, diagnosis, and treatment of Coronarvirus Disease 2019 (COVID 19). A Review. JAMA 2020;324:8.

2. Anwar A, Ramos-Bascon N, Crerar-Gilbert A, et al. A specialised cardiorespiratory team approach in the intensive care management of COVID-19 patients: benefit on mortality, diagnosis and management. Clin Med (Lond) 2021;21:101-6.

3. Brodsky MB, Levy MJ, Jedlanek E, et al. Laryngeal injury and upper airway symptoms after oral endotracheal intubation with mechanical ventilation during critical care: A systematic review. Crit Care Med 2018;46:2010-7.

4. Chao TN, Harbison SP, Braslow BM, et al. Outcomes after tracheostomy in COVID-19 patients. Ann Surg 2020;272:e181-6.

5. Oliver CM, Campbell M, Dulan O. Appearance and management of COVID-19 laryngo-tracheitis: two case reports. F1000Res 2020;9:310.

6. Minambres E, Buron J, Ballesteros MA. Tracheal rupture after endotracheal intubation: a literature systematic review. Eur J Cardiothorac Surg 2009;35;1056-62.

7. Wali A, Rizzo V, Bille A. Pneumomediastinum following intubation in COVID-19 patients: a case series. Anaesthesia 2020;75:1076-81.

8. Mangel T, Madden BP. Acute tracheal tear - A potential cause of spontaneous pneumomediastinum in patients with COVID19. Monaldi Arch Chest Dis 2021;91:1852.

9. Madden BP, Datta S, McAnulty GR. Tracheal granulation tissue after percutaneous tracheostomy treated with Nd:Yag laser: three cases. J Laryngol Otol 2001;115:743-4.

10. Touman A, Stratakos G. Long-term complications of tracheal intubation. In: R. Hakan Erbay, editor. Tracheal Intubation. IntechOpen; 2018.

11. Bacon J, Patterson C, Madden BP. Indications and interventional options for non-resectable tracheal stenosis. J Thorac Dis 2014;6:258-70.

12. RECOVERY Collaborative Group, Horby P, Lim WS, et al. Dexamethasone in hospitalized patients with Covid-19. N Engl J Med 2021;384:693-704.

13. Marty-Ane CH, Picard E, Jonquet O, Mary H. Membraneous tracheal rupture after endotracheal intubation. Ann Thorac Surg 1995;60:1367-71.

14. Ozcan A, Balci C, Aksoy S. Upper airway injury caused by gum elastic bougie. Int J Case Rep Images 2017;8:439-43.

15. Arndt G, Cambray A, Tomasson J. Intubation bougie dissection of tracheal mucosa and intratracheal airway obstruction. Anesth Analg 2008;107:603-4. 